\title{
Influence of low-gravity level on crystal growth in floating zone
}

\author{
Bin Xiong ${ }^{1}$ and Wen-Rui Hu \\ Institute of Mechanics, Chinese Academy of Sciences, Beijing 100080, People's Republic of China
}

Received 26 November 1992; manuscript received in final form 22 June 1993

\begin{abstract}
The influence of low gravity level on crystal growth in the floating zone, which involves thermocapillary convection, phase change convection, thermal and solutal diffusion, is investigated numerically by a finite element method for the silicon crystal growth process. The velocity, temperature, concentration fields and phase change interfaces depending on heating temperature and growth rates are analyzed. The influence of low gravity level on the concentration is studied especially. The results show that the non-uniformities of concentration are about $10^{-3}$ for growth rate $v_{\mathrm{p}}=5.12 \times 10^{-8} \mathrm{~m} / \mathrm{s}, 10^{-2}$ for $v_{\mathrm{p}}=5.12 \times 10^{-7} \mathrm{~m} / \mathrm{s}$ and relatively larger for larger growth rate in the gravity level $g=0-9.8 \mathrm{~m} / \mathrm{s}^{2}$. The thermocapillary effect is strong in comparison with the Bridgman system, and the level of low gravity is relatively insensitive for lower growth rates.
\end{abstract}

\section{Introduction}

There are different systems for crystal growth in which the convection will influence the quality of growth crystals. For example, the quality of crystal produced by the Bridgman-Stockbarger method on the ground is mainly affected by buoyancy-driven convection; therefore, the low gravity environment level is important for the better quality of crystal growth if the gravity level is minimized. It seems that this conclusion does not hold for the floating zone system which involves the free surfaces. In addition to the effect of buoyancy, another indispensable factor is surface tension, which may play an important role depending on the microgravity level in the floating zone system.

The gravity levels, from $10^{-2} g_{0}$ to $10^{-6} g_{0}$, may be obtained by different apparatuses [1], where $g_{0}$ is $9.8 \mathrm{~m} / \mathrm{s}^{2}$, the gravity on the ground. In order to evaluate these apparatuses, Rosenberger and co-workers [2] recently investigated numerically the effects of residual acceleration on dopant distributions during directional solidification in an ideal Bridgman-Stockbarger model simplified from the work of Chang and Brown [3]. Their results show that the lateral non-uniformity in composition is sensitive to the gravity level, and a steady background level of $10^{-6}$ times that of normal gravity can be tolerated for growth rates of several microns per second. This is a significant conclusion which can be used to appreciate the feasibility of space infrastructure for microgravity research. However, it will be an interesting question whether this conclusion also holds for other crystal growth systems, and whether the gravity level can be tolerated for the dopant non-uniformity in the floating zone system.

In our previous work [4,5], in which we have studied the thermal and solutal convection, diffusion and directional solidified interfacial shapes in the floating zone crystal growth process under zero gravity condition, we used the formulation in the following sections and the "up-wind" FEM method [6] to examine the extent of dopant non-uniformity in the model of the floating zone on gravity level from 0 to $1 g_{0}$. Comparisons are made between the results of the previous work [2,3] and those of the present paper.

${ }^{1}$ On leave to Department of Materials Science and Engineering, University of Wisconsin, Madison, Wisconsin, USA. 


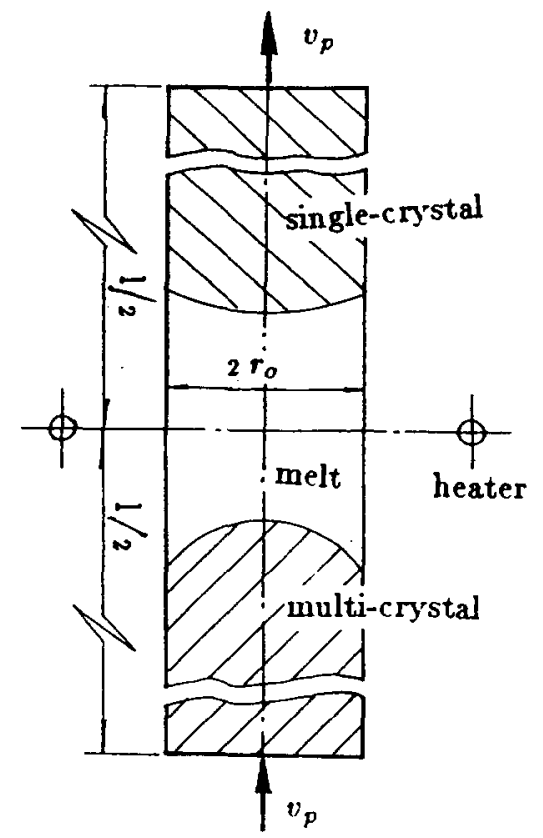

Fig. 1. Schematic diagram of floating zone system model.

\section{Model}

The physical model in the present paper analyzes the thermal diffusion in the solid region together with convections and diffusions in the liquid (melt) region while the cylinder pulled, as shown in fig. 1. To construct a complete model of full floating zone, three regions should be involved, that is, the liquid bridge region of molten silicon, the solid region of lower feed rod and the solid region of the upper single-crystal rod. Convection in the liquid region is assumed to be axisymmetric and steady. The plane $z=0$ in the cylindrical coordinate system is fixed in the heater plane, which is considered to be a thermal radiation ring. The single crystal and the feed rods have the same diameter $r_{0}$ and move with the same rate of $v_{\mathrm{p}}$ upward in $z$-direction. Free surface deformation is ignored for simplification, and the radius of the liquid bridge, $r_{0}$, is constant.

The governing equations include mass conservation, momentum conservation, energy conservation and diffusion equation under the suitable boundary conditions, the same as the ones in ref. [7] for a steady process. Dimensionless parameters are introduced as follows:

$$
\begin{aligned}
& \zeta=\frac{z}{r_{0}}, \quad \xi=\frac{r}{r_{0}}, \quad U=\frac{u}{u_{0}}, \quad W=\frac{w}{u_{0}}, \quad P=\frac{p}{\rho u_{0}^{2}}, \\
& \Theta_{i}=\frac{T_{i}-T_{\mathrm{m}}}{T_{0}-T_{\mathrm{m}}} \quad(i=1,2), \quad \Theta=\frac{T-T_{\mathrm{m}}}{T_{0}-T_{\mathrm{m}}}, \quad C=\frac{c}{c_{\mathrm{s}}},
\end{aligned}
$$

where the subscript 0 denotes the typical value; $T_{0}$ and $T_{\mathrm{m}}$ are the heating temperature and melting point, respectively; $(u, w)$ is the flow velocity in $(r, z)$ direction; $p, T$ and $c$ are the pressure, temperature and concentration in the liquid bridge, respectively; the subscripts 1 and 2 denote the value in the single crystal region and the feed region, respectively; the constant $c_{\mathrm{s}}$ is the concentration of the feed rod. The characteristic velocity $u_{0}$ remains to be determined. The appropriate characteristic velocity 
in the thermocapillary problem may be the characteristic velocity $u_{0}=\left|\sigma_{\mathrm{T}}\right|\left(T_{0}-T_{\mathrm{m}}\right) / \mu$ driven by the gradient of surface tension, where $\sigma_{\mathrm{T}}$ and $\mu$ are the gradient of surface tension and the viscosity, respectively. In this case the equations may be written as follows [5]:

$$
\mathrm{Pe}_{i} \frac{\partial \Theta_{i}}{\partial \zeta}=\frac{\partial^{2} \Theta_{i}}{\partial \zeta^{2}}+\frac{\partial^{2} \Theta_{i}}{\partial \xi^{2}}+\frac{1}{\xi} \frac{\partial \Theta_{i}}{\partial \xi} \quad(i=1,2),
$$

for the regions of the solid rods, and

$$
\begin{aligned}
& \frac{\partial U}{\partial \xi}+\frac{U}{\xi}+\frac{\partial W}{\partial \zeta}=0, \\
& \operatorname{Re}\left(U \frac{\partial U}{\partial \xi}+W \frac{\partial U}{\partial \zeta}\right)=-\operatorname{Re} \frac{\partial P}{\partial \xi}+\frac{\partial^{2} U}{\partial \zeta^{2}}+\frac{\partial^{2} U}{\partial \xi^{2}}+\frac{1}{\xi} \frac{\partial U}{\partial \xi}-\frac{U}{\xi^{2}}, \\
& \operatorname{Re}\left(U \frac{\partial W}{\partial \xi}+W \frac{\partial W}{\partial \zeta}\right)=\operatorname{Bo} \Theta-\operatorname{Re} \frac{\partial P}{\partial \zeta}+\frac{\partial^{2} W}{\partial \zeta^{2}}+\frac{\partial^{2} W}{\partial \xi^{2}}+\frac{1}{\xi} \frac{\partial W}{\partial \xi}, \\
& \operatorname{Ma}\left(U \frac{\partial \Theta}{\partial \xi}+W \frac{\partial \Theta}{\partial \zeta}\right)=\frac{\partial^{2} \Theta}{\partial \zeta^{2}}+\frac{\partial^{2} \Theta}{\partial \xi^{2}}+\frac{1}{\xi} \frac{\partial \Theta}{\partial \xi}, \\
& \operatorname{Pe}_{\mathrm{s}}\left(U \frac{\partial C}{\partial \xi}+W \frac{\partial C}{\partial \zeta}\right)=\frac{\partial^{2} C}{\partial \zeta^{2}}+\frac{\partial^{2} C}{\partial \xi^{2}}+\frac{1}{\xi} \frac{\partial C}{\partial \xi}
\end{aligned}
$$

for the region of the liquid bridge. The dimensionless boundary conditions are

$$
\begin{aligned}
& \xi=0, \quad \zeta \notin\left(S_{2}(0), S_{1}(0)\right): \quad \frac{\partial \Theta_{1}}{\partial \xi}=0, \quad \frac{\partial \Theta_{2}}{\partial \xi}=0, \\
& \xi=1, \quad \zeta \notin\left(S_{2}(1), S_{1}(1)\right): \quad-\frac{\partial \Theta_{i}}{\partial \xi}=\mathrm{Bi}_{i}\left[\left(\Theta_{i}+T_{\mathrm{m}}^{*}\right)^{4}-T_{0}^{* 4}\right] f(\zeta) \quad(i=1,2), \\
& \zeta=S_{i}(\xi), \quad \xi \in(0,1): \quad \Theta_{i}=0 \quad(i=1,2), \\
& \zeta= \pm L_{i}, \quad \xi \in(0,1): \quad \Theta_{i}=\Theta_{\infty_{i}} \quad(i=1,2),
\end{aligned}
$$

for the regions of the solid rods, and

$$
\begin{aligned}
\xi=0, \quad \zeta \in\left(S_{2}(0), S_{1}(0)\right): & \frac{\partial U}{\partial \xi}=0, \quad \frac{\partial W}{\partial \xi}=0, \quad \frac{\partial \Theta}{\partial \xi}=0, \quad \frac{\partial C}{\partial \xi}=0 \\
\xi=1, \quad \zeta \in\left(S_{2}(1), S_{1}(1)\right): \quad & U=0, \quad \frac{\partial W}{\partial \xi}=-\frac{\partial \Theta}{\partial \zeta}-\delta_{\mathrm{s}} \frac{\partial C}{\partial \zeta} \\
& \partial C / \partial \zeta=0 \\
& \left.-\partial \Theta / \partial \xi=\mathrm{Bi}\left[\left(\Theta+T_{\mathrm{m}}^{*}\right)^{4}-T_{0}^{* 4}\right)\right] f(\zeta), \\
\zeta=S_{1}(\xi), \quad \xi \in(0,1): \quad U= & \frac{\epsilon_{1} V_{\mathrm{p}_{1}}\left(\mathrm{~d} S_{1} / \mathrm{d} \xi\right)}{1+\left(\mathrm{d} S_{1} / \mathrm{d} \xi\right)^{2}}, \\
W= & \frac{\epsilon_{1} V_{\mathrm{p}_{1}}}{1+\left(\mathrm{d} S_{1} / \mathrm{d} \xi\right)^{2}}-V_{\mathrm{p}_{1}},
\end{aligned}
$$




$$
\begin{array}{ll}
\Theta=0, & \\
\partial C / \partial n=\operatorname{Pe}_{\mathrm{s}} V_{\mathrm{p}_{n}}\left(1-k_{0}\right) C, & \\
\zeta=S_{1}(\xi), \quad \xi \in(0,1): \quad & U=\frac{\epsilon_{2} V_{\mathrm{p}_{2}}\left(\mathrm{~d} S_{2} / \mathrm{d} \xi\right)}{1+\left(\mathrm{d} S_{2} / \mathrm{d} \xi\right)^{2}}, \\
& W=\frac{\epsilon_{2} V_{\mathrm{p}_{2}}}{1+\left(\mathrm{d} S_{2} / \mathrm{d} \xi\right)^{2}}-V_{\mathrm{p}_{2}}, \\
\Theta & =0, \\
& \partial C / \partial n=-\mathrm{Pe}_{\mathrm{s}} V_{\mathrm{p}_{n}}\left(C-C_{\mathrm{s}}\right),
\end{array}
$$

for the region of the liquid bridge, where $\zeta=S_{1}(\xi)$ and $\zeta=S_{2}(\xi)$ are the melting boundary and the solidification boundary of phase change interfaces, respectively. The dimensionless Stefan conditions become

$$
\frac{\partial \Theta_{i}}{\partial n}-\delta_{i} \frac{\partial \Theta}{\partial n}= \pm \frac{\mathrm{St}_{i}\left|V_{p+}\right|}{\left[1+\left(\mathrm{d} S_{i} / \mathrm{d} \xi\right)^{2}\right]^{1 / 2}} \quad(i=1,2)
$$

The following dimensionless controlling parameters have been introduced:

$$
\begin{aligned}
& \mathrm{Re}=\frac{r_{0} u_{0}}{\nu}, \quad \mathrm{Ma}=\frac{r_{0} u_{0}}{\alpha}, \quad \mathrm{Bo}=\frac{\beta g r_{0}^{2}\left(T_{0}-T_{\mathrm{m}}\right)}{\nu u_{0}}, \quad \mathrm{Pe}_{i}=\frac{v_{\mathrm{p}_{i}} r_{0}}{\alpha_{i}}, \\
& \delta_{i}=\frac{K}{K_{i}}, \quad \mathrm{St}_{i}=\frac{\Delta H_{i} \rho r_{0} u_{0}}{K_{i}\left(T_{0}-T_{\mathrm{m}}\right)}, \quad T_{\mathrm{m}}^{*}=\frac{T_{\mathrm{m}}}{T_{0}-T_{\mathrm{m}}}, \\
& T_{0}^{*}=\frac{T_{0}}{T_{0}-T_{\mathrm{m}}}, \quad V_{\mathrm{p}_{i}}=\frac{v_{\mathrm{p}_{i}}}{u_{0}}, \quad \mathrm{Bi}_{i}=\frac{f_{0} r_{0} \epsilon_{i}^{*} \sigma^{*}\left(T_{0}-T_{\mathrm{m}}\right)^{3}}{K_{i}}, \\
& \mathrm{Bi}=\frac{f_{0} R_{0} \epsilon^{*} \sigma^{*}\left(T_{0}-T_{\mathrm{m}}\right)^{3}}{K}, \quad \mathrm{Pe}_{\mathrm{s}}=\frac{r_{0} u_{0}}{D}, \quad \delta_{\mathrm{s}}=\frac{\sigma_{\mathrm{s}} c_{\mathrm{s}}}{\sigma_{T} \Delta T} \quad(i=1,2)
\end{aligned}
$$

where $\alpha$ is thermal diffusivity, $K$ is thermal conductivity, $\Delta H$ is the latent heat of phase change, $\epsilon^{*}$ is the emissivity, $\sigma^{*}$ is the Stefan-Boltzmann constant, $D$ is the dopant diffusivity in the melt, $k_{0}$ is the dopant segregation coefficient, $V_{\mathrm{p}_{n}}=V_{\mathrm{p}} /\left[1+(\mathrm{d} S / \mathrm{d} \xi)^{2}\right]^{0.5}$, and $n$ is the normal unit vector to interfaces which directs to melt region. The solution in the three regions will satisfy the matching conditions at the phase change interfaces $\zeta=S_{1}(\xi)$ and $\zeta=S_{2}(\xi)$. The Stefan conditions, together with other boundary conditions, are used to determine the shapes of interfaces. As usual, we introduced stream function $\psi$ and vorticity $\Omega$, and the equations in the liquid bridge may then be expressed in relations of stream function-vorticity $[4,5]$.

The non-uniformity of concentration in the molten zone at the solidification interface to be the lateral range in concentration is defined as

$$
\lambda=\left(C_{\max }-C_{\min }\right) / \bar{C},
$$

where $C_{\max }, C_{\min }$ and $C$ are maximum, minimum and average values at the solidification interface respectively. 
The main object of the present work is to study the influence of low gravity level on the melt flow, solute distribution, and especially the non-uniformity of concentration. The influence of gravity magnitude $g / g_{0}$ and the pulling rate $V_{\mathrm{p}_{i}}$ are analyzed also. The phase change interfaces can be obtained by an iteration method of pseudo-transient process, of which the Stefan conditions are treated as time-dependent equations.

\section{Numerical simulation}

The governing equations with appropriate boundary conditions are solved by the so-called "up-wind" finite element method [5,6]. A quadrilateral iso-parametric element is employed. The phase change interfaces are calculated by a "pseudo time-dependent" method [5]. The numerical simulation is applied especially for the crystal growth of silicon, of which the thermophysical properties are given in refs. $[5,8]$. A diluted solute of small concentration is considered. The segregation coefficient $k_{0}$ is assumed to be 0.1 in the present paper. In order to obtain steady results, we use a smaller value of temperature difference $\Delta T=T_{0}-T_{\mathrm{m}}=10 \mathrm{~K}$, where the melting point $T_{\mathrm{m}}=1688 \mathrm{~K}$. Smaller temperature differences at the ends of both solid rods are given to form the appropriate melting zone, therefore, we adopt $T_{\infty_{i}}=1643 \mathrm{~K}$, the radii of the rods are the same $(5 \mathrm{~cm})$, and the ampoule length $l$ is $40 \mathrm{~cm}$.

According to our previous conclusions [5], the results show that the thermocapillary convection may act as a strong mixer, and the dimensionless solutal concentrations in the liquid region are much larger than the dimensionless concentration value in the multi-crystal region $C_{0}=1$. As the intensity of the thermocapillary convections is much larger than the intensity of the forced flow of pulling, the concentration values in the liquid bridge are always nearly uniform no matter how the gravity level is. This means that the thermocapillary convection plays a similar role as the buoyancy-driven convection in the model without free surface under large-parameter conditions, such as Bridgman crystal growth shown in refs. [2,3]. Two different gravity acceleration directions are considered in the present paper: (1) one is opposite to the growth direction; (2) the other is parallel to the growth direction.

Figs. 2, 3 and 4 exhibit the flow patterns, temperature distributions and concentration fields for three conditions.

Fig. 2 illustrates the case in different low gravity levels when the growth rate $v_{\mathrm{p}}=5.12 \times 10^{-7} \mathrm{~m} / \mathrm{s}$ and the direction of gravity acceleration is opposite to the pulling velocity. The magnitude of the growth rate, $v_{\mathrm{p}}=5.12 \times 10^{-7} \mathrm{~m} / \mathrm{s}$, is large enough due to the given thermal conditions. It could be seen that the melting zone shifts to the upper side. The upper flow cell is so large that it dominates almost the whole zone, even in the case of $g=0$, as shown in fig. $2 a$. Because the buoyancy-driven convection is counter-clockwise in this case, the upper flow cell is tensified and the bottom one is weakened by the buoyancy-driven convection. In general, the larger the gravity acceleration, the larger the magnitude of maximum flow velocity in the buoyancy dominant case. All the flow patterns and temperature distributions are similar when the gravity acceleration is smaller than $9.8 \times 10^{-2} \mathrm{~m} / \mathrm{s}^{2}$ for the same pulling rate. Additionally, the isotherms display inverse S-shape in the case of larger gravity levels. The dopant non-uniformity is so small, about $(3-6) \times 10^{-2}$, that the non-uniformity appears nearly at the place of the axis. The shapes of melting and solidification interfaces are different in different gravity environments. The solidification interface and the melting interface becomes convex, and the buoyancy-driven flow moves towards the solidification interface near the free surface under the condition of larger gravity.

Figs. 3 and 4 show the results in the case of smaller growth rates, $v_{\mathrm{p}}=5.12 \times 10^{-8} \mathrm{~m} / \mathrm{s}$, and the gravity direction being either opposite or parallel to the growth direction. In both cases, the symmetrical patterns on the heating plane, when $g=0$, change into asymmetrical ones when $g \neq 0$. The flow is tensified by buoyancy force. When the gravity acceleration direction is parallel to the pulling velocity, the solidification interface becomes more convex with the increment of gravity, but the melting interface 

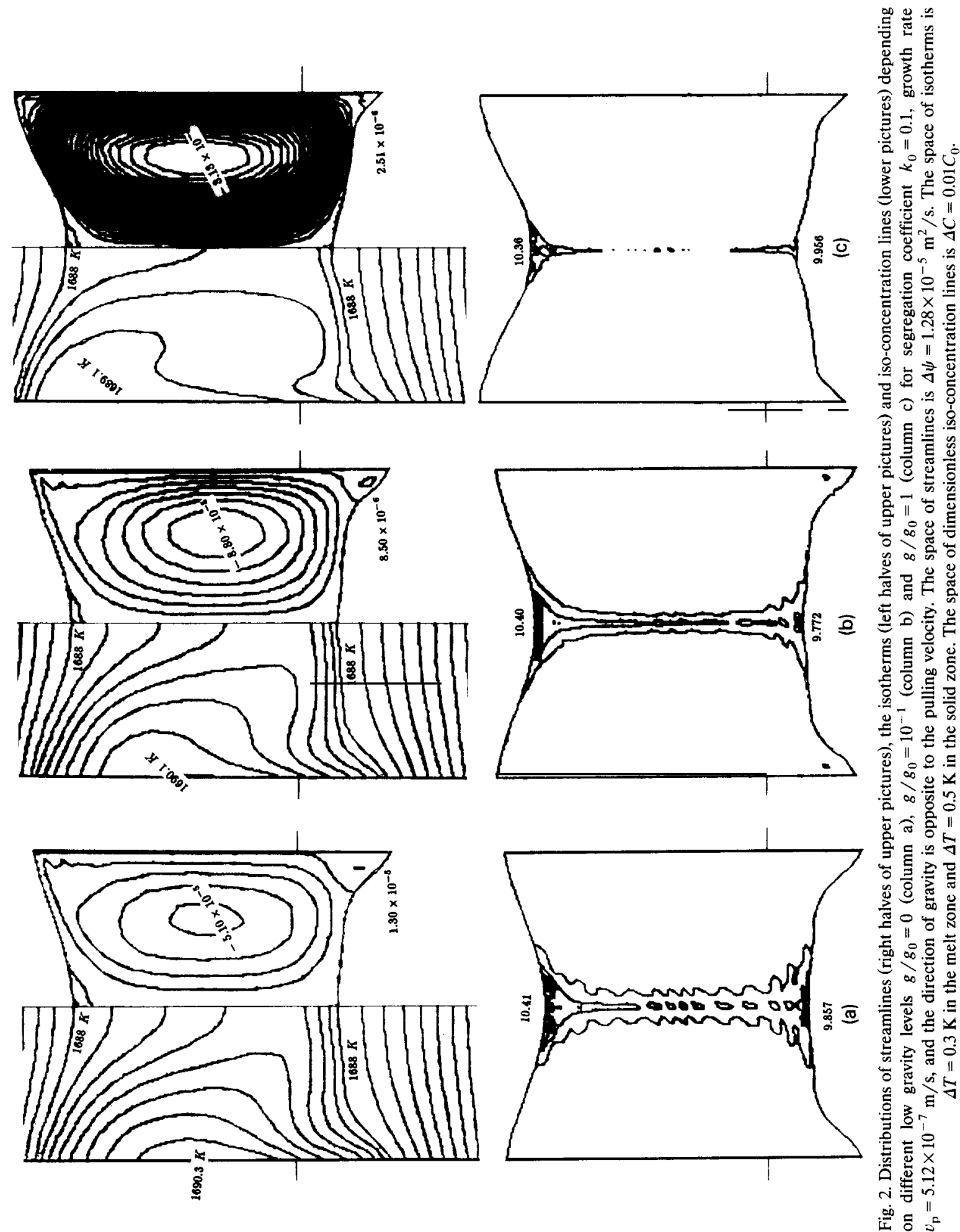

僯 

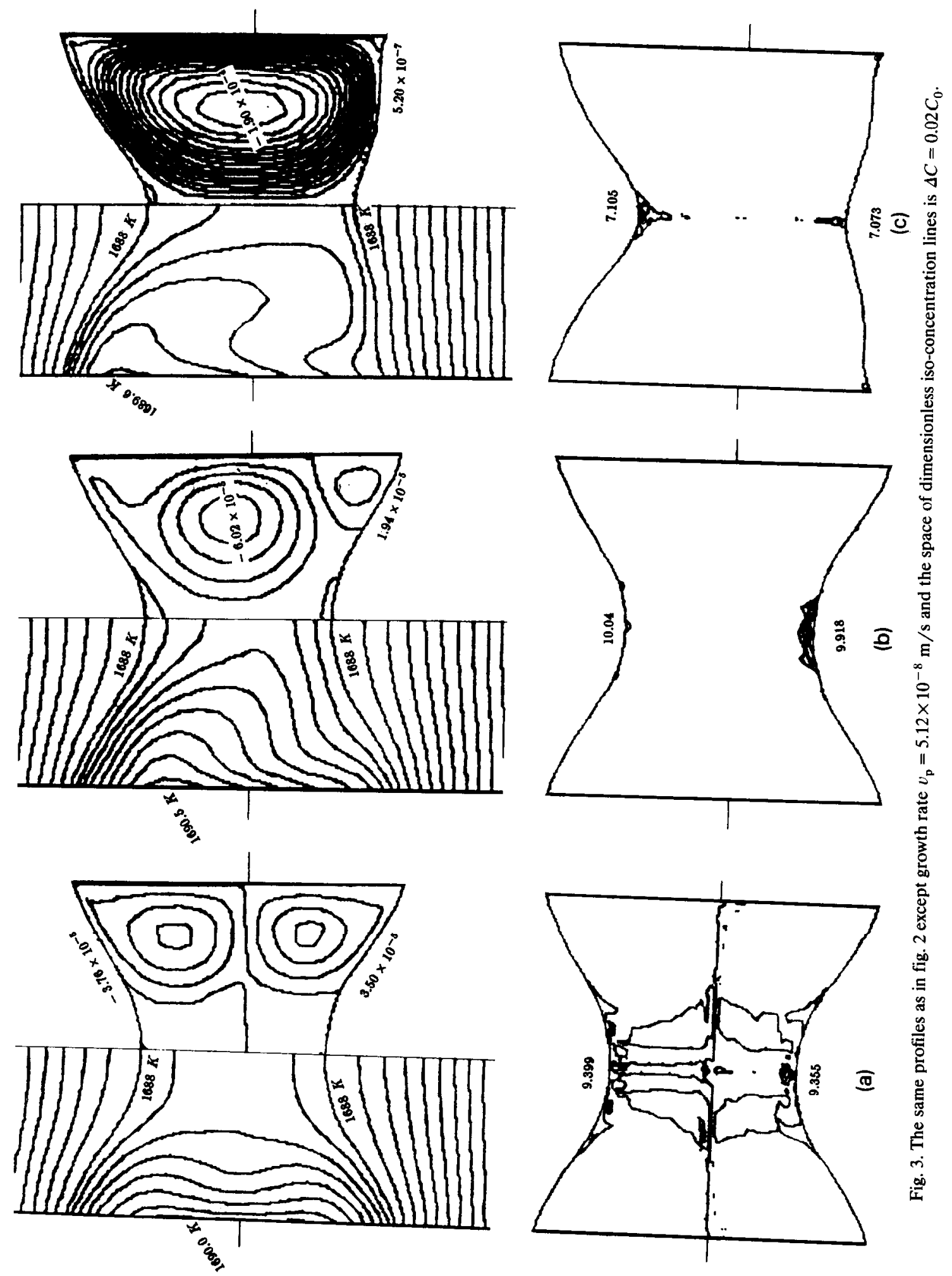

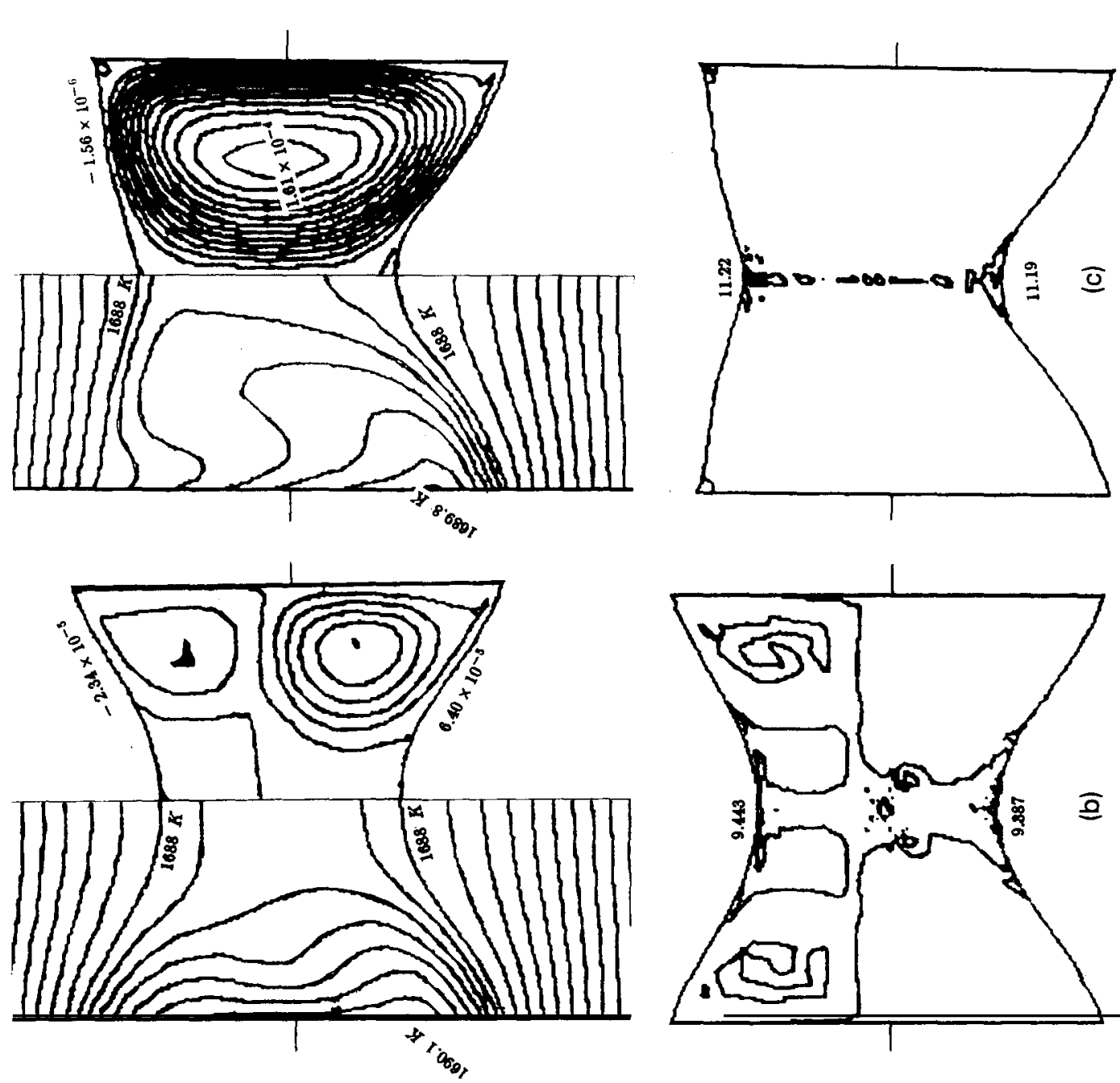

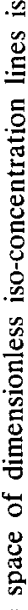
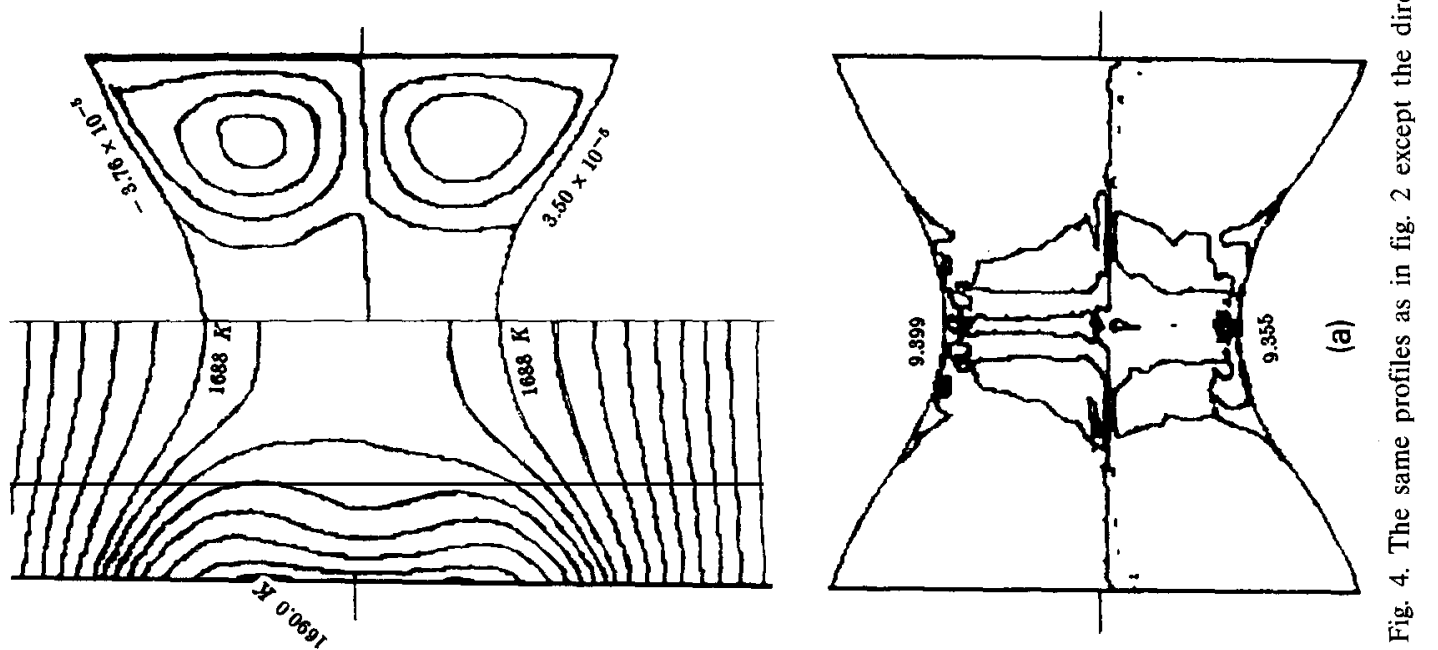


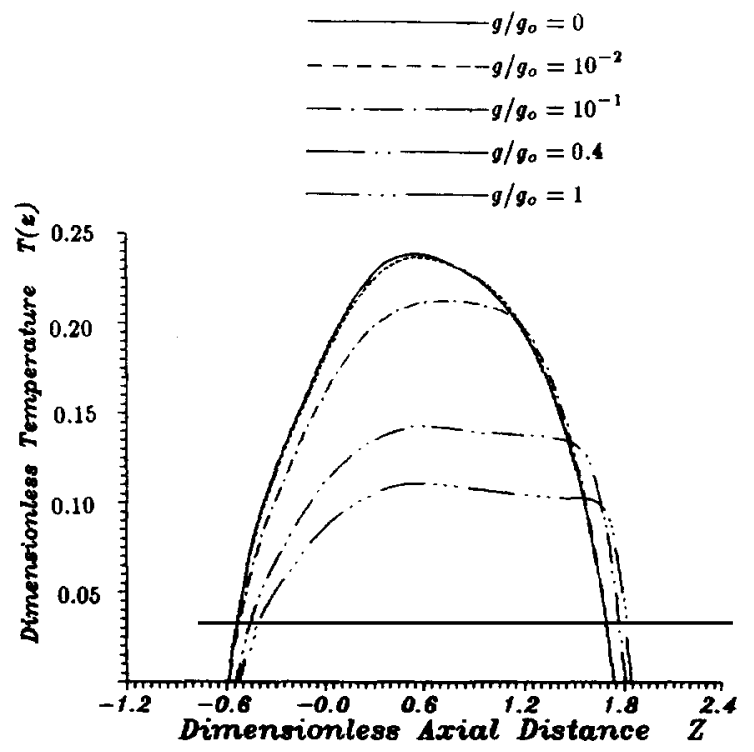

Fig. 5. Longitudinal temperature profiles on the free surface of melting zone depending gravity level for $v_{\mathrm{p}}=5.16 \times 10^{-7} \mathrm{~m} / \mathrm{s}$, $k_{0}=0.1$ and the direction of gravity opposite to the pulling velocity.

becomes relatively flat. The magnitude of the non-uniformities in the two cases is about $(2-4) \times 10^{-3}$, which is smaller than the ones in the case of larger growth rate.

Figs. 5-7 are the temperature distributions on the free surface for different low-gravity levels. Generally, the maximum of the temperature in the molten liquid bridge deduces with an increment of the magnitude of gravity. It is shown that the convection driven by buoyancy indeed plays an important role in thermal distribution.

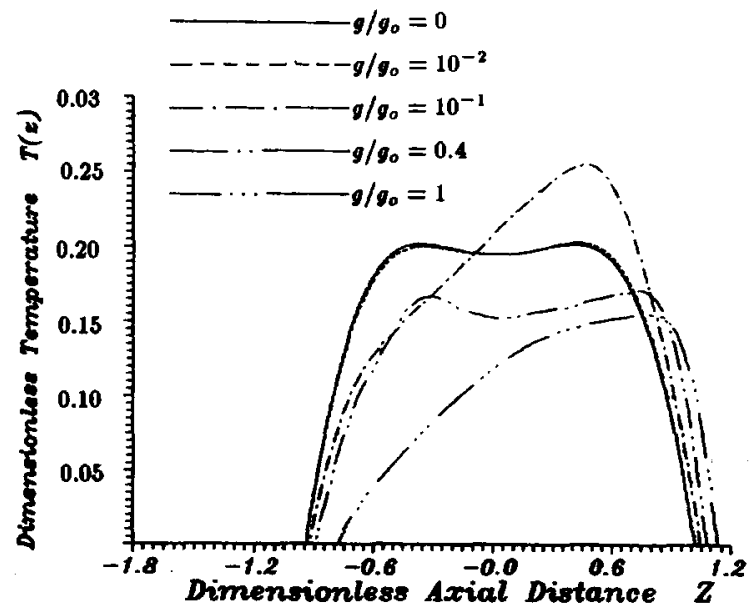

Fig. 6. Longitudinal temperature profiles on the free surface of melting zone depending gravity level for $v_{\mathrm{p}}=5.16 \times 10^{-8} \mathrm{~m} / \mathrm{s}$, $k_{0}=0.1$ and the direction of gravity opposite to the pulling velocity. 


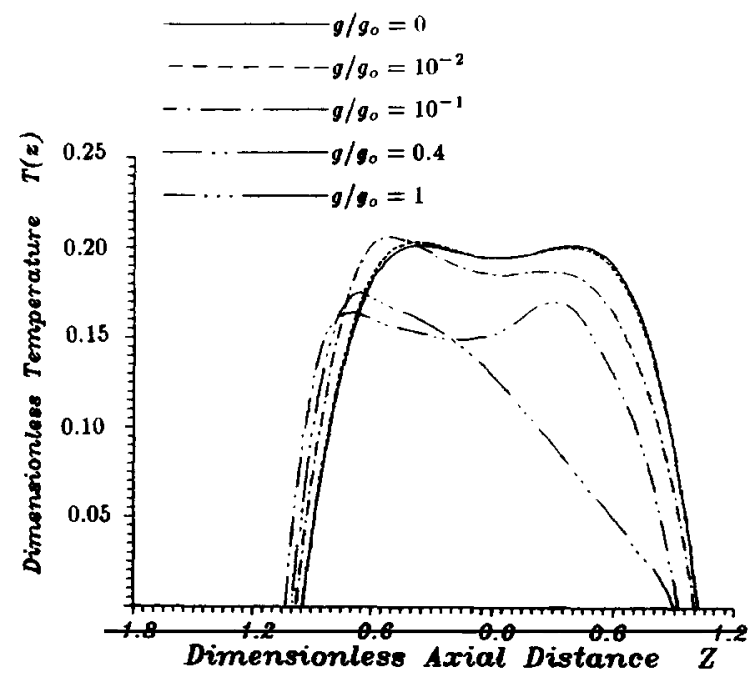

Fig. 7. Longitudinal temperature profiles on the free surface of melting zone depending gravity level for $v_{\mathrm{p}}=5.16 \times 10^{-8} \mathrm{~m} / \mathrm{s}$, $k_{0}=0.1$ and the direction of gravity parallel to the pulling velocity.

Figs. 8 and 9 display the radial variations of dopant concentration at the solidification interface and the axial variations along symmetrical axis for different gravity conditions and fixed growth rate $v_{\mathrm{p}}=5.12 \times 10^{-7} \mathrm{~m} / \mathrm{s}$. The results show that there are segregations in the two directions, even though the convections make the dopant distributions smooth enough. It is also noticed that the radial variations of dopant concentration at the solidification interface are not monotonic and the "ripple peak" appears at the place of the dimensionless radial distance $R=0.1-0.15$. The case is distinct from that in the Bridgman model which has no free surface.

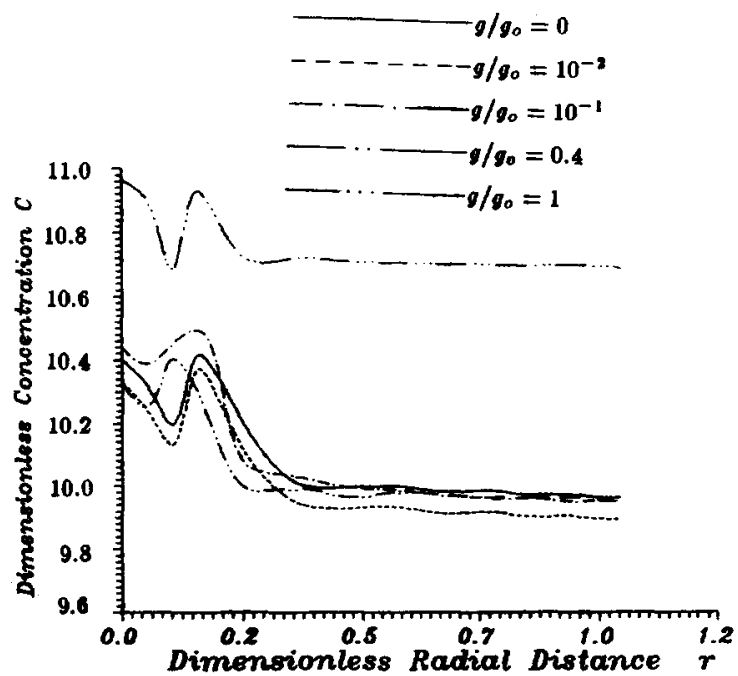

Fig. 8. Concentration profiles in the radial direction at the solidification interface depending on gravity level for $v_{\mathrm{p}}=5.16 \times 10^{-7}$ $\mathrm{m} / \mathrm{s}, k_{0}=0.1$ and the direction of gravity opposite to the pulling velocity. 


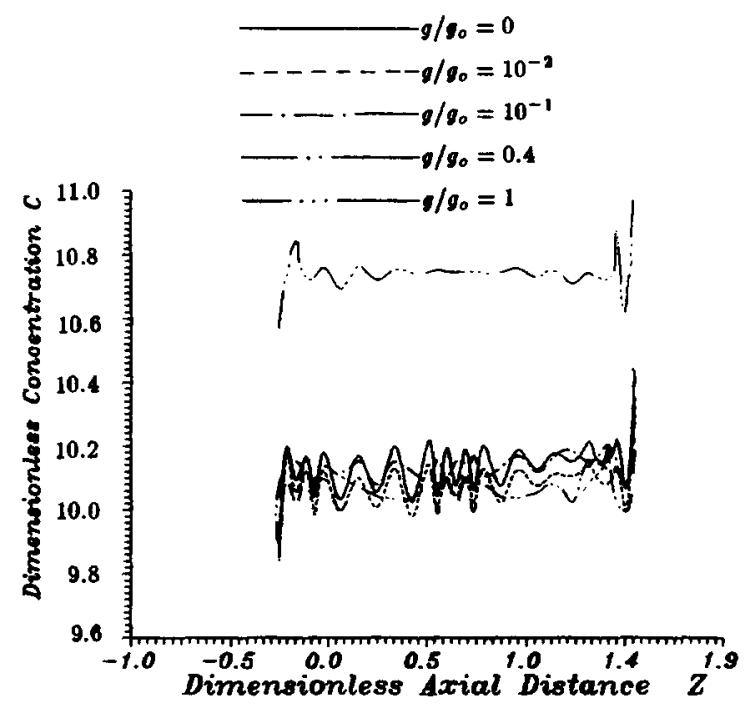

Fig. 9. Concentration profiles along the central axis depending on gravity levels for $v_{\mathrm{p}}=5.16 \times 10^{-7} \mathrm{~m} / \mathrm{s}, k_{0}=0.1$ and the direction of gravity opposite to the pulling velocity.

The radial variations of dopant concentration at the solidification interface and the longitudinal variations along the axes of symmetry are shown in figs. 10 and 11 for different gravity conditions and fixed growth rate $v_{\mathrm{p}}=5.16 \times 10^{-8} \mathrm{~m} / \mathrm{s}$. The nearly constant distributions of profile lines may be explained by the fact that the uniform forced flow of the pulling rod is concealed by the strong thermocapillary-driven and the buoyancy-driven convections.

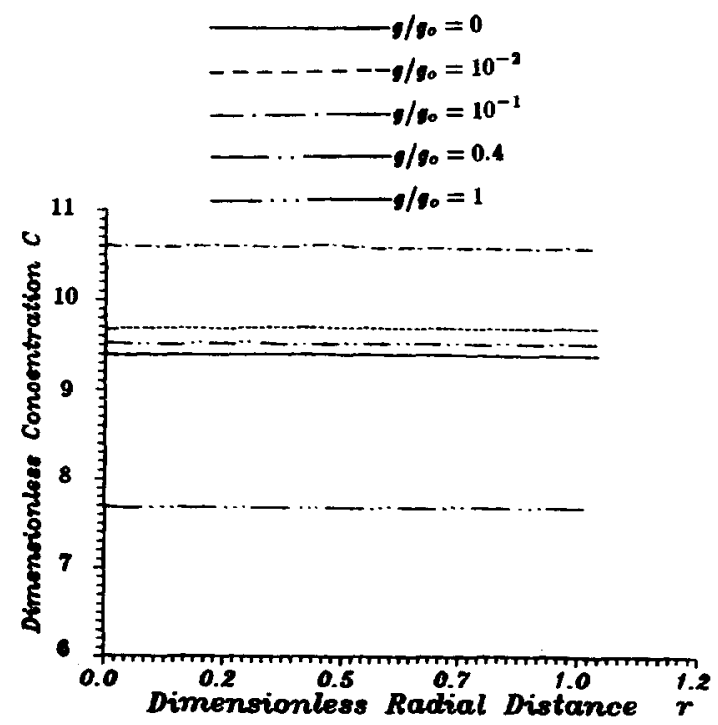

Fig. 10. Concentration profiles in the radialdirection-at-the-solidification interface depending on gravity levels for $v_{\mathrm{p}}=5.16 \times 10^{-8}$ $\mathrm{m} / \mathrm{s}, k_{0}=0.1$ and the direction of gravity opposite to the pulling velocity. 


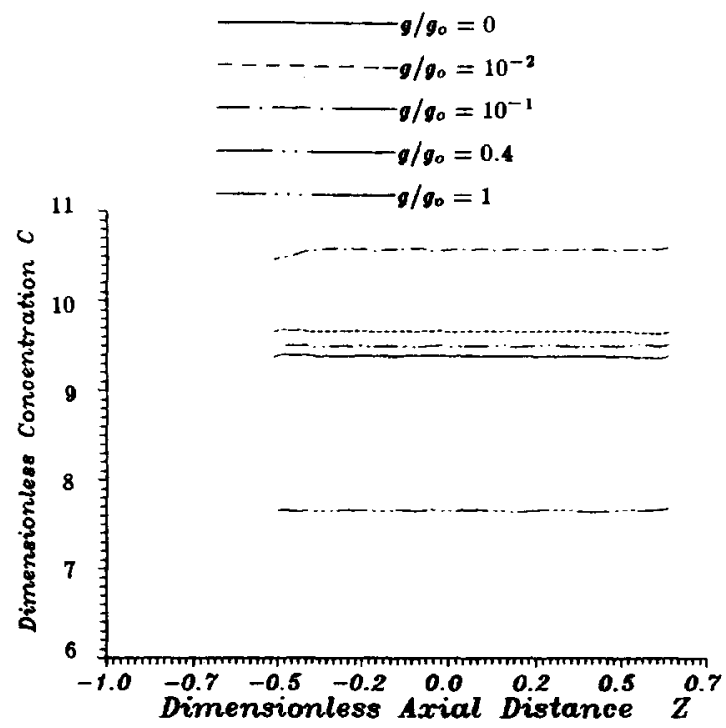

Fig. 11. Concentration profiles along the central axis under different gravity levels for $v_{\mathrm{p}}=5.16 \times 10^{-8} \mathrm{~m} / \mathrm{s}, k_{0}=0.1$ and the direction of gravity opposite to the pulling velocity.

The effect of gravity level on the dopant non-uniformity is shown in fig. 12 . The relationship between the gravity level and the non-uniformity is nonlinear, similar to the results in ref. [3]. The maximum of the dopant non-uniformity emerges at $\mathrm{g} / \mathrm{g}_{0}=0.2$ for growth rate $v_{\mathrm{p}}=5.12 \times 10^{-8} \mathrm{~m} / \mathrm{s}$ and $\mathrm{g} / \mathrm{g}_{0}=0.08$ for $v_{\mathrm{p}}=5.12 \times 10^{-7} \mathrm{~m} / \mathrm{s}$. But the non-uniformities in the present analyses are much smaller than the ones in refs. [2,3], although the typical scale is much larger here, because the thermocapillary-driven convection is so strong in the floating zone that the flow exceeds the "first critical" flow in the case of the buoyancy-driven convection as in refs. [2,3]. It can be seen from the results in refs. [2,3] and the present paper that the dopant non-uniformity $\lambda$ becomes large if the magnitude of the growth rate is increased to be comparable with the convection velocity in the molten zone of the liquid bridge. As the growth rate is much smaller than the maximum convection velocity, about $1 \times 10^{-2}$ to $3 \times 10^{-2}$ in the present paper,

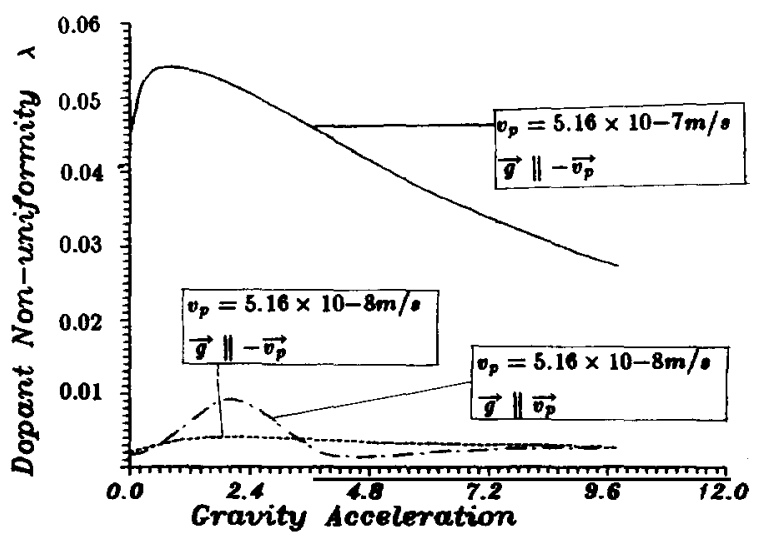

Fig. 12. Dopant non-uniformity at the solidification interface as a function of gravity level for different pulling velocities and segregation coefficient $k_{0}=0.1$. 
we can conclude that it is unnecessary to worry about the tolerance for the effect of microgravity levels, produced by various microgravity tools, on the uniformity of dopant concentrations in the floating zone crystal growth system for the case of reasonable growth rate and typical scale.

\section{Discussion and conclusions}

A finite element method is applied to calculate the effect of the convection of different gravity levels $\left(g=0-9.8 \mathrm{~m} / \mathrm{s}^{2}\right)$ on the dopant concentration distributions, flow patterns, temperature distributions and the phase change interfaces for the steady process of crystal growth in the floating zone. The so-called "up-wind" technique is employed to overcome the difficulty induced by the strong convection. It is proved that the technique is successful to handle this problem in which the complex flow is stronger than the buoyancy-driven convection denoted in ref. [2].

The numerical results show that the distributions of dopand concentration in the floating zone crystal growth system are more uniform in the case of the present paper than those in the crystal growth system without free surfaces. The non-uniformities are much smaller than those in ref. [2], although the rod diameter in the present work is 10 times larger than the one in ref. [2], and the dynamic Bond number may be larger than $B_{d}=13.9$, which shows that the effect of thermocapillary force on fluid flow is strong enough in comparison with the effect of the buoyancy-driven convection in ref. [2]. It is concluded that the floating zone crystal growth can tolerate much larger gravity. The relationship between the gravity acceleration and the dopant non-uniformity is nonlinear, and the "critical" gravity $g_{\text {cr }}$ in the profiles of the $g-\lambda$ relationship is different for different growth rate conditions.

The gravity level has an influence on the temperature distribution as well as on the shape of the interface. The effect of the gravity is to make the solidification interface convex and the melting interface flat if the gravitational direction is opposite to the pulling velocity, but convexity only appears near the crosspoint of the symmetrical axis and the melting interface. The melting interface is relatively convex when the direction of gravity is parallel to the direction of growth.

\section{References}

[1] B. Feuerbacher, H. Hamacher and R.J. Naumann, Materials Sciences in Space (Springer, Berlin, 1988).

[2] J.I.D. Alexander, J. Ouazzani and F. Rosenberger, J. Crystal Growth 97 (1989) 285.

[3] Ch.J. Chang and R.A. Brown, J. Crystal Growth 63 (1983) 343.

[4] R.R. You and W.R. Hu, IAF-91-395 (AIAA, 1991).

[5] B. Xiong and W.R. Hu, J. Crystal Growth 125 (1992) 149.

[6] J.C. Heinrich, P.S. Huyakorn and O.C. Zienkiewicz, Intern. J. Numer. Methods Eng. 11 (1977) 131.

[7] B. Xiong, Z.M. Tang and W.R. Hu, Microgravity, Sci. Technol. 6 (1993) 131.

[8] C.W. Lan and Sindo Kou, J. Crystal Growth 108 (1991) 351.

[9] W.R. Hu and Z.M. Tang, Scientia Sinica A 33 (1990) 934. 